Nachteile haben wir bei unseren Untersuchungen Barbitalnatrium-Acetatpuffer bei $\mathrm{pH}$ 8,1 verwendet und damit gut reproduzierbare Ergebnisse erzielt.

Als Wasserstoffdonator für die Peroxydasereaktion scheint das bereits früher von WATSON (12) verwendete o-Tolidin am besten geeignet (Abb. 2). o-Dianisidin hemmt die Galaktoseoxydasereaktion kompetitiv, wie Fischer und ZAPF $(6,11)$ nachgewiesen haben (Abb. 2). Allerdings fanden andere Autoren bei Verwendung von o-Dianisidin lineare Eichkurven $(7,10)$. Bei Verwendung von o-Kresol $(6,11)$ sind die Eichkurven zu flach, um genaue Bestimmungen zu ermöglichen (Abb. 2).

Die störende Wirkung verschiedener Faktoren im Blut war bereits früher aufgefallen. VERDIER und HJELM (10) stellten Eichkurven im Serum der untersuchten Personen auf, um die störende Wirkung der verschiedenen Faktoren zu eliminieren. Rотн und Mitarbeiter (8) haben keine näheren Angaben über die Art der hemmenden Substanzen im Blut gemacht. Enteiweißung wurde mit Bariumhydroxyd-Zinksulfat durchgeführt. Diese Enteiweißung ist jedoch sehr umständlich und wird durch die erforderliche genaue Einstellung der Reagenzienlösung kompliziert. Fischer und $\operatorname{ZAPF}_{\text {APF }}(6,11)$ haben darauf hingewiesen, daß die Harnsäure und reduziertes Glutathion wahrscheinlich als Wasserstoffdonatoren für die Peroxydasereaktion dienen können. Auch Harnstoff und Kreatinin wird von diesen Autoren eine deutliche Hemmwirkung zugeschrieben. In dem von uns verwendeten Testansatz störten jedoch lediglich Harnsäure, Glutathion und sehr hohe Konzentrationen von Ascorbinsäure. Frscher und ZApr (11) eliminierten die störenden Substanzen durch Inkubation der Proben mit Ionenaustauschern. Auch dieses Verfahren ist für Routineuntersuchungen zu umständlich. Ein wesentlicher Vorteil der von uns verwendeten Enteiweißung mit Quecksilberacetat-Perchlorsäure liegt daher in der einfachen Durchführbarkeit. Durch Quecksilberacetat werden die Enzymreaktionen nicht beeinflußt, die störenden Substanzen mit Ausnahme von Ascorbinsäure jedoch vollständig eliminiert.

Bei Vorgehen nach der Testvorschrift der Fa. Worthington sind gegenüber unserer Modifikation zwei zusätzliche Pipettierungen erforderlich. Daneben wurde der Harnsäure- und Glutathioneinfluß nicht berücksichtigt. Auch gegenüber den von anderen Autoren angegebenen Nachweisvorschriften $(6,7,8,10)$ zeichnet sich die von uns durchgeführte Modifikation besonders durch den geringeren Zeitaufwand aus; daneben wurden alle störenden Faktoren ausgeschaltet.

\title{
Literatur
}

1. Förster, H. und H. Mehnert, Methoden der Kohlenhydratbestimmungen. Hdb. Kinderheilkunde Bd. II/1, S. 754. SpringerVerlag, Bcrlin-Heidelberg-New York (1966). - 2. Watson, D., Analyt. Chem. 5, 260 (1963). - 3. FörSTER, H., Unveröffentl. Ergebn. - 4. Avigad, D., D. Amaral, C. Asenio und B. L. HorECKER, J. biol. Chemistry 237, 2736 (1962). - 5. CoOper, J. A. D., W. Smith, M. Bacita und M. Medina, J. biol. Chemistry 234, 445 (1959). - 6. FISCHER, W. und J. ZAPF, Hoppe-Seylers Z. physiol. Chem. 337, 186 (1964). - 7. Ford, J. D. und J. C. Haworth, Clin. Chem. (New York) 11, 1002 (1964). - 8. Roth, H., S. SEgaL und D. Bertor, Analyt. Biochem. (New York) 10, 32 (1965). -
9. MrChaelis, L., Biochem. Z. 234, 139 (1931). - 10. Verdier, C. H. DE und M. HJELM, Clin. chim. Acta (Amsterdam) 7, 742 (1962). - 11. FrsCHER, W. und J. ZAPF, Hoppe-Seylers Z. physiol. Chem. 339, 54 (1964). - 12. WAtson, D., Analyt. Biochem. (New York) 3, 131 (1962). - 13. Axelrod, J., J. Pharmacol. exp. Therapeut. 138, 28 (1962). - 14. Kutrer, D., Aerztl. Laborat. 7, 175 (1961). - 15. Scrmidr, F. H., Methoden der Blut- und Harnzuckerbestimmung unter besonderer Berücksichtigung enzymatischer Verfahren, S. 76. Fortschr. Diabetesforsch., Georg Thieme Verlag, Stuttgart (1963). - 16. Schultherss, F. R., Helvet. med. acta 33, 59 (1966).

Dr. H. Förster

6 Frankfurt/Main, Ludwig-Rehn-Str. 14

\section{Bestimmung der Oxydation von Östradiol zu Östron im menschlichen Organismus mit Hilfe von Östradiol-17 $\alpha-T$}

\author{
Von M. WenzeL und K.-U. BLuM \\ Auls dem Pbysiologisch-chemischen Institut (Direktor: Prof. Dr. Dr. E. Scbütte) und der I. Medizinischen Klinik \\ (Direktor: Prof. Dr. Dr. l. c. H. Frh. v. Kress) der Freien Universität Berlin
}

(Eingegangen am 20. Februar 1967)

Es wird eine Methode beschrieben, mit der das Mindestausmaß der biologischen Oxydation von Oestradiol zu Oestron im Gesamtorganismus gemessen werden kann. Von dem injizierten Oestradiol-17 $\alpha-\mathrm{T}$ wird durch die Oestradioldehydrogenase das Tritium auf Pyridinnucleotide übertragen und letztlich zu Tritiumwasser verbrannt. Die Zunahme der Konzentration des Tritiumwassers im Gesamtkörperwasser gilt als Maßstab für die Oxydation des Steroids. Die Zeitabhängigkeit der Oestradioloxydation bei einigen Versuchspersonen wird angegeben. Bei Patienten ohne pathologischen Leberbefund werden innerhalb von 24 Stdn. mindestens $50-70 \%$ des injizierten Oestradiols oxydiert und nur 3\% im Urin als $17 \alpha$-T-Steroid ausgeschieden.

A method is described for measuring the oxidation of oestradiol to oestrone in the whole organism. The tritium of injected oestradiol-17 $\alpha-\mathrm{T}$ is transferred to pyridine nucleotides by oestradiol dehydrogenase and finally metabolised to tritiated water. The increase in the concentration of tritiated water in the total body water is a measure of the oxidation of the steroid. The time course of oestradiol oxidation is given for some experimental persons. In patients with no pathological liver condition at least $50-70 \%$ of the injected oestradiol is oxidised within $24 \mathrm{hrs}$. and only $3 \%$ is excreted in the urine as $17 \alpha-\mathrm{T}$ steroid. 
Die biologische Wirksamkeit der „klassischen“ Oestrogene Oestradiol, Oestron und Oestriol verhält sich im Allen-Dorsy-Test annähernd wie 100:10:1 (1). Daher müßte die Kenntnis des Ausmaßes der Oxydation von Oestradiol zu Oestron - und der Oestradiolderivate $\mathrm{zu}$ Oestronderivaten - gleichzeitig einen Einblick in das Mindestmaß der biologischen Inaktivierung des wirksamsten weiblichen Sexualhormons vermitteln. Als Hauptorgan für den Abbau des Oestradiols wird die Leber angesehen $(1,2)$. Störungen der Leberfunktion sollen daher auch Störungen im Stoffwechsel der Oestrogene nach sich ziehen $(1,3)$. Daher müßte, falls es gelingt, den Umfang der Oxydation des Oestradiols zu dem um eine Zehnerpotenz weniger wirksamen Oestron zu bestimmen, geklärt werden können, ob Beziehungen zwischen der Leberfunktion und der Oestradiol-Inaktivierung durch Oxydation bestehen. Aus diesem Grunde soll zunächst über die ,in vivo"-Oxydation am C-Atom 17 des Östradiols bei lebergesunden Patienten berichtet werden.

\section{Methodik}

\section{Pringi力 des Verfabrens}

Das Erfassen der Oxydation der 17-Hydroxy- zur $17-$ Ketogruppe beim Oestradiol sowie gleichzeitig bei seinen 17-Hydroxy-Derivaten ist fast immer unmöglich und bereitet selbst „in vitro“ große Schwierigkeiten. Dies schon allein deswegen, weil nicht alle Abbauwege des Oestradiols bekannt sind und daher auch nicht alle Dcrivate des 17-Hydroxy- bzw. 17-Ketosteroides nachzuweisen sind (4).

Frühere Versuche an Ratten hatten gezeigt, daß mit Hilfe eines 17-Hydroxysteroides, das am $\mathrm{C}_{17}$-Atom mit Tritium markiert ist, die Oxydation von 17-Hydroxy- zu 17-Ketosteroiden innerhalb des Organismus erfaßt werden kann $(5,6)$. Durch die Oxydation der Hydroxyzur Ketogruppe am $\mathrm{C}_{17}$-Atom wird das Tritium aus dem Molekül entfernt. Dies geschieht mit Hilfe der 17Hydroxy-Steroid-Dehydrogenase, die DPN oder TPN als Coenzym benötigt. Bei dieser Oxydo-Reduktion lagern die Coenzyme das Tritium unter Reduktion zu DPN-T bzw. TPN-T an und schleusen ihrerseits das Wasserstoffisotop in die Atmungskette ein, in der das Tritium zu Wasser verbrannt wird:

\begin{tabular}{|c|c|c|}
\hline & Ostradiol-17 $\alpha-\mathrm{T}+$ & \\
\hline$\frac{\text { Steroid }}{\text { Dehydrogenase }} \rightarrow$ & Östron $+\begin{array}{c}\text { DPN-T } \\
(\text { TPN-T })\end{array}$ & Atmungskette \\
\hline
\end{tabular}

Das aus DPN-T oder TPN-T gebildete Tritiumwasser vermischt sich nach kurzer Zeit mit dem Gesamtkörperwasser. Das Auftreten von einem Molekül Tritiumwasser (HTO) zeigt damit die Oxydation von einem Moleküil Oestradiol an (5).

'Da die reduzierten Coenzyme ihr Tritium nicht nur in die Atmungskette leiten sondern auch zu Synthesen verbrauchen können, wird mit diesem Verfahren immer nur ein Mindestmaß der Oxydation erfaßt, allerdings unabhängig davon, in welchen Organen sie stattfindet. So läßt sich bei Oestradiol die Mindestinaktivierung dieses Hormones zu dem biologisch wesentlich schwächer wirksamen
Oestron messen, ohne eine Vielzahl von Mctaboliten zu isolieren Zur Bestimmung der Menge an Tritium im Gesamtkörperwasser mißt man von cinem Aliquot die spezifische Aktivität und rechnet auf den Gesamtgehalt an Körperwasser um. Diese Möglichkcit ist der entscheidende Vorteil des geschilderten Prinzips. Bei allen anderen bckannten Methoden zur Umsatzbestimmung ist der Gehalt an einem bestimmten Metaboliten in einzelnen Körperbestandteilen nicht als repräsentativ für den Gehalt im gesamten Organismus anzusehen. Diese Voraussetzung trifft allein für die Verteilung radioaktiven Wassers im Gesamtkörperwasser zu. Um das reine Wasser von eventuell vorhandenen anderen radioaktiven Substanzen abzutrennen, ist eine vorherige Destillation des Wassers aus einer Probe, z. B. Harn oder Blut, notwendig. Noch einfacher läßt sich durch Aktivitätsmessung des Atemwassers die spezifische Aktivität des Körperwassers feststellen $(5,6,7)$.

\section{Arbeitsweisc}

Jede Versuchsperson erhielt $148 \mu \mathrm{g}$ Oestradiol-17 $\alpha-\mathrm{T}$ (5) mit ciner Aktivität von $99 \mu \mathrm{C}$, in Propylenglykol/0,9-proz. NaCl-Lösung (1:1) gelöst, i. m. injiziert. Hier wurde einerseits eine Hormonmenge angewendet, die sich im physiologischen Bereich hlt (1), ä andererseits cine Menge von Tritium, die unterhalb der gesetzlichen Freigrenze für den Umgang mit Tritium markierten Materials liegt (8). Wenn auch dic Inkorporation von einigen $100 \mu \mathrm{C}$ Tritium in bezug auf die Strahlenbelastung ungefährlich ist - zur Körperwasserbestimmung werden $z$. B. $500-2000 \mu \mathrm{C}$ verwendet $(8,9)-$ sollte hier aus prinzipiellen Gründen diese gesetzliche Freigrenze nicht überschritten werden.

Die spez. Aktivität des Tritiumwassers aus dem Blut oder dem Urin wurde nach der Gefrierdestillation der jeweiligen alkalisierten Flüssigkeiten im „Tri Carb" gemessen. Die Gefrierdestillation wurde quantitativ durchgeführt, um Fehlcr durch Isotopie-Effekte zu vermeiden. Die Blutentnahme erfolgte durch Venenpunktion. Um Urinproben zu gewinnen, wurde Katheterurin über die Dauer von $15 \mathrm{Min}$. vor bis $15 \mathrm{Min}$. nach dem jeweiligen Meßpunkt gesammelt. Hierdurch wurden Urinproben gemessen, bei denen der Urin gerade die Nieren verlassen hat und cine Verdünnung durch vorher in der Blase angesammelten schwächer aktiven Urins nicht eintreten konnte.

\section{Bereclsnung}

Das Ausmaß der Oxydation von Oestradiol zu Oestron und HTO wurde folgendermaßen berechnet: Die spez. Akt. in zpm/ml Körperwasser, die sich aus der inj. Aktivität und dem Körperwassergehalt (im Alter von 51-60 Jahren bei Frauen 50,5\% und bei Männern 57,4\% der Körpergewichte (10)) ergab, wurde gleich $100 \%$ gesetzt. Die gefundene spez. Akt. in den destillierten und den gemessenen Körperflüssigkeiten in $\mathrm{zpm} / \mathrm{m} l$ wurde dann in Prozent zu der inj. Aktivität angegeben nach der Formel:

\section{zpm $_{\text {HTO }}$ pro $\mathrm{m} l \mathrm{Körperwasser}$}

zpminj. Ocstr. pro $\mathrm{ml}$ Körperwasser $100=\%$ Mindestoxydation des Oestradiols

Versucbspersonen

M. Sch., weiblich, 71 Jahre; klinische Diagnose: Perniciöse Anämie, atrophische Gastritis, Hiatushernie. Befunde: Leber und Milz o. B., Elektrophorese unauffällig. Gallenfarbstoff o. B., Thromboplastinzeit $100 \%$.

A. M., männlich, 64 Jahre; klinische Diagnose: Altersdiabctes, Encephalomalazie. Befunde: Leber und Milz o. B., Elektrophorese: außer einer geringen $\gamma$-Globulinverminderung unauffällig. Gallenfarbstoffe $\mathrm{o}$. B.

L. O., weiblich, 61 Jahre; klinische Diagnose: Hypertonie, Encephalomalazie. Befunde: Leber und Milz o. B., Elektrophorese unauffällig. Gallenfarbstoffe o. B., Transaminasen im Serum nicht erhöht. Thromboplastinzeit $76 \%$.

O.F., weiblich, 79 Jahre; klinische Diagnose: Hypertonic, Herzinsuffizienz. Befunde: Leber und Milz o. B., Elektrophorese unauffällig. Gallenfarbstoffe o. B.

R. D., männlich, 38 Jabre; klinische Diagnose: Chron. Cholecystitis, Cholelithiasis, Gastritis. Befunde: Leber und Milz 
nicht vergrößert. Druckschmerz i. rechten Oberbauch. Elektrophorese unauffällig. Brompthaleintest negativ. Gallenfarbstoffe zum Zeitpunkt des Testes o. B., Transaminasen im Serum nicht erhöht. Thromboplastinzeit $100 \%$.

\section{Ergebnisse und Diskussion}

Die Abbildung 1 zeigt die Zeitabhängigkeit der Mindestoxydation von Oestradiol-17 $\alpha-\mathrm{T}$ zu Oestron bei drei weiblichen und einer männlichen Versuchsperson.

Danach ergibt sich in diesen 4 Fällen bis zu $14 \mathrm{Stdn}$. nach der Injektion ein langsames Ansteigen der HTO-

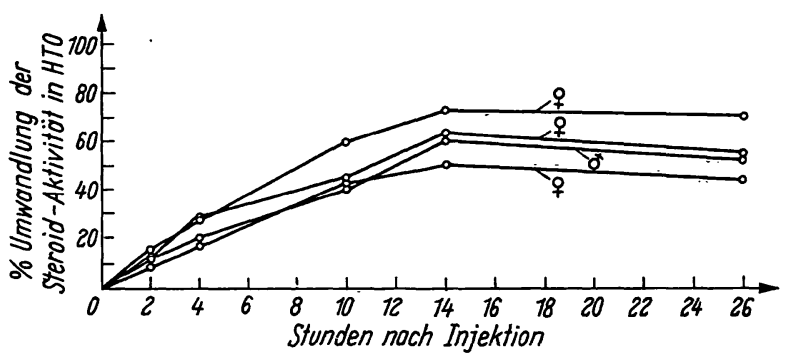

Abb. 1

Zeitabhängigkeit der Oxydation von Oestradiol-17 $\alpha-T$ zu HTO

Konzentration im Körperwasser und damit der Werte für die Oestradiol-Oxydation. Nach $14 \mathrm{Stdn}$. ist der höchste Wert der Oestradiol-Oxydation mit $62 \pm 10 \%$ der injizierten Dosis erreicht. Danach fällt der HTOSpiegel im Körperwasser mit einer Halbwertzeit von 3-6 Tagen ab, was im Bereich der in der Literatur angegebenen Werte für die Ausscheidung von TritiumWasser beim Menschen (mittlere biologische Halbwertzeit 9 Tage) liegt $(8,9)$. Das Auftreten des Plateaus nach 14 Stdn. zeigt gleichzeitig, daß eine weitere Erhöhung der spez. Aktivität des Körperwassers nicht stattfindet, also nach $14 \mathrm{Stdn}$. kein weiteres Oestradiol mehr oxydiert wird.

Dieses Ergebnis und die Tatsache, daß bei diesen 4 Fällen nur etwa $62 \%$ des injizierten Oestradiols als HTO erscheinen, ließe sich am einfachsten so erklären, daß ein großer Teil der applizierten Oestradiolmenge in den ersten $14 \mathrm{Stdn}$. als Oestradiol oder als am $\mathrm{C}_{17}$-Atom nicht oxydiertes Oestradiolderivat (z. B. Sulfat- oder Glucuronsäureester) ausgeschieden wurde.

Um diese Tritium-markierten Produkte zu erfassen, wurde bei einem der Patienten (M. Sch.) von den destillierten Harnproben die Aktivität im Rückstand gemessen. Nach den in Tabelle 1 wiedergegebenen Ergebnissen war die Aktivität, die einem Rücks̄tand von $1 \mathrm{~m} l$ Harn entsprach, bei den zu den verschiedenen Zeitpunkten gewonnenen Proben etwa gleichgroß, so daß ein Mittelwert gebildet werden konnte. Aus dem durchschnittlichen Harnvolumen von 1,25 l/24 Stdn. und der Durchschnittsaktivität (im Rückstand) von $5 \times 10^{3} \mathrm{zpm} / \mathrm{m} l$ Harn ergibt sich, daß in $24 \mathrm{Stdn}$. nur etwa $2,8 \mu \mathrm{C}$ oder $2,8 \%$ der injizierten Aktivität in Form von $17 \alpha$-TSteroid ausgeschieden worden sind. Diese Zahl stimmt gut mit entsprechenden Werten von Fishman (10) überein, der bei zwei Frauen 3,6\% bzw. 2,9\% der injizierten Aktivität von Oestradiol-17 $\alpha$-T in den Harn- rückständen (Harnsammlung in den ersten 24 Stdn. nach der Injektion) finden konnte.

Nach diesen Ergebnissen sind also innerhalb der ersten 24 Stdn. 30-35\% der injizierten Aktivität des Oestradiol-17 $\alpha-\mathrm{T}$ weder als HTO nachweisbar, noch als Ester mit dem Urin ausgeschieden worden. Das kann daran liegen, daß dieser Anteil des Oestradiols tatsächlich noch nicht oxydiert worden ist oder daran, daß ein Teil der Tritiumaktivität $z$ war auf DPN oder TPN übertragen, aber statt zu HTO verbrannt, zu Synthesen verbraucht worden ist. Nach früheren Untersuchungen kann aber dieser Anteil nicht sehr groß sein. In vitroVersuche mit Leberschnitten, Diaphragma (11) sowie Ascitestumorzellen (12), bei denen intrazellulär DPN-T oder TPN-T erzeugt worden war, oder in vivo-Versuche mit Ratten nach Laktat-2-D-Gabe (13) zeigten nämlich einen 100 bis 1000 fach stärkeren Einbau der über die Coenzyme umgesetzten Wasserstoffisotope in Wasser als in anderen Zellbestandteilen. Aus den Untersuchungen von Fishiman und Mitarbeitern, die erstmals Oestradiol-17 $\alpha=T$, allerdings für Studien über die in vivo-Umwandlung Oestradiol-Oestron-Oestriol verwendeten (10), läßt sich das Ausmaß der OestradiolOxydation im Gesamtorganismus leider nicht errechnen und mit diesen Ergebnissen vergleichen, da die verabreichte Aktivität nur in Relativzahlen (c.p.m. = ipm) und nicht in absoluten Zahlen (zpm oder $\mu \mathrm{C}$ ) angegeben worden ist. Außerdem fehlt eine Gewichtsangabe der Versuchsperson, und somit auch eine Angabe des Körperwasseranteils.

Um zu überprüfen, ob die spezifische Aktivität des Körperwassers, bestimmt aus Urin oder aus Serum, zu identischen Werten für die Oestradiol-Oxydation führt, wurde bei 2 Versuchspersonen eine gleichzeitige Analyse in beiden Körperflüssigkeiten durchgeführt. Es ergaben sich zu den gleichen Zeitpunkten praktisch die gleichen Werte für die spezifische Aktivität des Körperwassers und damit für die Oestradioloxydation, wie aus Tabelle 1 $z u$ ersehen ist und zwar sowohl bei einer weiblichen als auch bei einer männlichen Versuchsperson. Diese

Tạb. 1

Zeitabhängigkeit der Tritium-Aktivität im Körperwasser aus Serum und aus Urin sowie der Steroid-17a-T-Ausscheidung im Urin

Den Patienten wurden je $98 \mu \mathrm{C}$ Oestradiol-17 $\alpha-\mathrm{T}$ i. v. injiziert und zu den angegebenen Zeiten Serum oder Urin gewonnen. Aus den Serumund Urin-Proben wurde reines Wasser durch Tieftemperatur-Destillation erhalten. Im Rückstand des Urin-Destillates wurde ebenfalls die Tritium-Aktivität gemessen (Patient M. Sch.) Mittelwert $\times$ Harnmenge in 24 Stdn. ergibt die Ausscheidung von nicht oxydiertem Steroid oder Steroid-Derivaten $\left(5 \cdot 10^{3} \mathrm{zpm} / \mathrm{ml} \times 1250 \mathrm{ml}=6,25 \cdot 10^{6} \mathrm{zpm}\right)$. Das entspricht einer Ausscheidung von $2,8 \mu \mathrm{C} / 24$ Stdn.

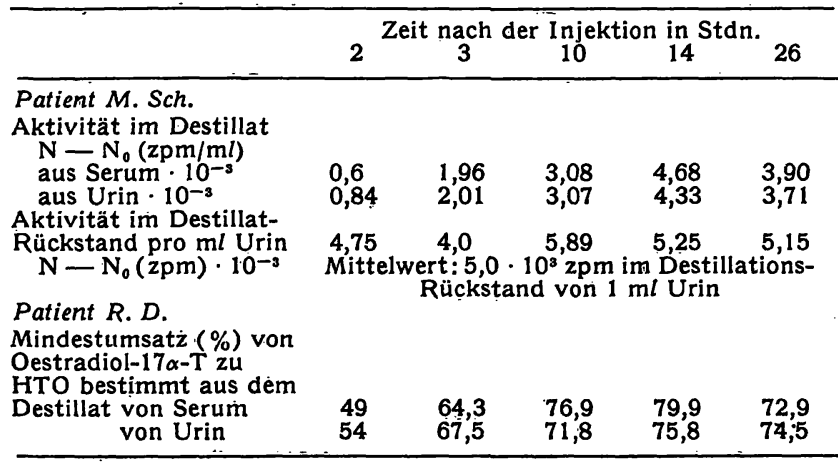


Beobachtung steht im Einklang mit MOORE (7), der eine Übereinstimmung der spezifischen Aktivität des Körperwassers feststellen konnte, das aus Urin, Blut und Atemwasser beim Menschen gewonnen wurde, denen HTO injiziert worden war. Danach hat sich beim Menschen ein Gleichgewicht des Tritiumgehaltes zwischen allen Körperflüssigkeiten spätestens nach etwa 1 Stunde eingestellt.

Durch das in Tabelle 1 dargestellte Ergebnis kann ferner eine bevorzugte Oxydation des Oestradiols durch das Nierenparenchym mit direkt anschließendem Austritt von HTO in den Urin vor Vermischen mit dem übrigen Körperwasser ausgeschlossen wërden, da dann der HTO- .
Spiegel im Urin höher sein müßte als im Serum. Aus den Werten für die männliche Versuchsperson in Tabelle 1 ergibt sich gegenüber den Fällen aus Abbildung 1 eine beschleunigte Oestradioloxydation, die dann auch zu einem höheren HTO-Spiegel im Körperwasser führte. Entsprechend mußte hier auch die beschleunigte Oestradioloxydation eine verminderte Ausscheidung von Oestradiol-17 $\alpha$-T oder von 17-Hydroxy-Derivaten festzustellen sein, was eine Untersuchung der Destillationsrückstände der Urinproben bestätigte.

Für fleißige Mitarbeit danken wir Fräulein Barbara HaNisCH und für materielle Unterstützung der Fa. Schering A. G., Berlin.

\title{
Literatur
}

1. Diczfalusy, E. und Cit. Lauritzen, Östrogene bei Menschen, Springer-Verlag, Berlin-Göttingen-Heidelberg (1961). - 2. RYAN, K. und L. ENGel, Endocrinology 52, 277 (1953). - 3. KAISER, E., W. Rindt und G. Oertel, Acta Hepato-Splenologica 12, 12 (1965). - 4. Breuer, H., R. KNUppen und M. Haupt, Nature (London) 212, 76 (1966). - 5. WeNZEL, M., H. KLEUCKer und E. P. SChulze, Z. Naturforsch. 21b, 1178 (1966). - 6. WENZEL, M. und E. KRAAS, Acta endocr., K'hvn. im Druck. - 7. MOORE, R.,
Health Physics 7, 161 (1962). - 8. Wenzel, M. und P. E. SCHulze, Tritium-Markierung, Walter de Gruyter \& Co., Berlin (1962): 9. Udekwu, F., D. Kozoll und K. Meyer, J. Nuclear Med. 4, 60 (1963). - 10. Fishiman, J., H. Bradlow, B. Zumoff, L. Hellman und T. Gallagher, Acta endocr. K'hvn 37, 57 (1961). - 11. Forster, D. und B. Bloom, J. biol. Chemistry 236, 2548 (1961). - 12. Wenzel, M., Habilitat. F. U. Berlin (1965). - 13. Hobermann, H., J. biol. Chemistry 233, 1045 (1958).

\section{Enzymatische Triglyceridbestimmungen bei Kindern}

\author{
Herrn Professor Dr. Dr. h. c. E. KLENK zum 70. Geburtstag \\ Von H. Wolf, M. Kochsiek-SChuster und H. LöHR ${ }^{1}$ ) \\ Aus der Universitäts-Kinderklinik" Göttingen (Direktor: Professor Dr. G. Joppicb)
}

(Eingegangen am 3. November 1966)

Die von Kreutz inaugurierte Methode der enzymatischen Bestimmung von freiem Glycerin und Serum-Triglyceriden wird anhand zahlreicher Beispiele in ihren Anwendungsmöglichkeiten in der.Pädiatrie erläutert. Eine eigene Ultramikromodifikation dieser Methode wird speziell für Untersuchungen des Stoffwechsels von freiem Glycerin und von intravenös zugeführten Triglyceriden bei Frühgeborenen ausgearbeitet.

Many examples from paediatrics are given of the use of KREUTz's method for the measurement of free glycerol and serum triglycerides. An ultramicro modification of this method is reported for the investigation of the metabolism of free glycerol and of intravenously administered triglycerides in premature children.

Seit mehreren Jahren wird den Neutralfetten des Serums auch im Bereich der pädiatrischen Forschung und Diagnostik zunehmend Beachtung geschenkt. Eine exakte Bestimmung der Serum-Triglyceride ist vor allem für dynamische Untersuchungen des Stoffwechsels in der Neugeborenenzeit $(1,2)$, zur Kontrolle von Lipid-Veränderungen in Abhängigkeit von der Ernährung $(3,4,5)$. und zu diagnostischen Zwecken erwünscht.

Chemische Verfahren zur Bestimmung des GlycerinAnteils der Triglyceride mit Perjodat $(6,7,8)$ erfordern neben einem beträchtlichen apparativen und zeitlichen Aufwand relativ große Blutmengen. Neutralfettbercchnungen als Differenz aus Esterfettsäuren und Lipoidfettsäuren (9) bzw. aus den Gesamtfettsäuren nach Mikrotitration (10) sind zwar für diagnostische Zwecke genau genug, jedoch für wissenschaftliche Untersuchun1) Teilweise vorgetragen auf dem 1. Weltfettkongreß, Hamburg 1964. gen der Dynamik von Neutralfettveränderungen kaum brauchbar, zumal auch hierbei der Bedarf an Serum für Untersuchungen bei Neugeborenen und Frühgeborenen $z u$ groß ist. Chylomikronenzählung (11) und Ultramikrobestimmung der Gesamtfette (12) lassen nur indirekte Aussagen über die Neutralfette zu und sind wiederum nur für dynamische Untersuchungen des postprandialen Ablaufs der Lipämie brauchbar (13). Hinsichtlich der Spezifität gebührt denjenigen Methoden der Vorzug, bei denen der Glycerinanteil der Triglyceride nach Hydrolyse en zy matiscl bestimmt wird. Enzymatische Glycerinbestimmungen sind von WIELAND (14), HAGEN und HAGEN (15), KREUTZ $(16,17)$, bzw. EgGSTEIN und Kreutz (18) sowie Garland und Randle (19) angegeben worden.

Wir bedienten uns für Untersuchungen des Fettstoffwechsels im Säuglingsalter, bei Frühgeborenen in den ersten Lebenstagen und bei Kindern mit verschiedenen 